\title{
Direct visualization of magnetic vortex pinning in superconductors
}

\author{
Mariela Menghini, ${ }^{1}$ R. B. G. Kramer, ${ }^{1}$ A. V. Silhanek, ${ }^{1}$ J. Sautner, ${ }^{2}$ V. Metlushko, ${ }^{2}$ K. De Keyser,${ }^{1}$ J. Fritzsche, ${ }^{1}$ \\ N. Verellen, ${ }^{1}$ and V. V. Moshchalkov ${ }^{1}$ \\ ${ }^{1}$ Nanoscale Superconductivity and Magnetism and Pulsed Fields Group, Institute for Nanoscale Physics and Chemistry (INPAC), \\ K. U. Leuven Celestijnenlaan 200 D, B-3001 Leuven, Belgium \\ ${ }^{2}$ Department of Electrical and Computer Engineering, University of Illinois, Chicago, Illinois 60607, USA
}

(Received 7 July 2008; revised manuscript received 15 December 2008; published 1 April 2009)

\begin{abstract}
We study the vortex structure in a $\mathrm{Pb}$ film deposited on top of a periodic array of ferromagnetic square microrings by combining two high-resolution imaging techniques: Bitter decoration and scanning Hall-probe microscopy (SHPM). The periodicity and strength of the magnetic pinning potential generated by the square microrings are controlled by the magnetic history of the template. When the square rings are in the magnetized dipolar state, known as the onion state, the strong stray field generated at the domain walls prevents the decoration of vortices. SHPM images show that the stray field generated by the dipoles is much stronger than the vortex field, in agreement with the results of simulations. Real-space vortex imaging has revealed that in the onion state, the corners of the square rings act as effective pinning centers for vortices.
\end{abstract}

DOI: 10.1103/PhysRevB.79.144501

PACS number(s): 74.25.Qt, 74.78.Db, 74.78.Na

\section{INTRODUCTION}

The most attractive hallmark of superconductivity from the technological stand point is the ability to carry electrical current without losses. Unfortunately, this fragile dissipationless state can be destroyed as a consequence of the motion of quantum flux lines (vortices) under the action of the Lorentz force induced by an applied electric current. During the last decades considerable effort has been devoted to prevent this vortex motion by anchoring the vortices using several methods. The vast majority of the research so far has been focused on studying core and electromagnetic pinning mechanisms. ${ }^{1-4}$ The former is related to the condensation energy saved when a vortex locates at the position of the pinning center, whereas the latter originates when the supercurrents flowing around the vortices in a distance of the order of the penetration length, $\lambda$, are deformed due to the presence of the pinning sites. In general, an enhanced vortex core pinning is achieved when the size of the pinning site is similar to the superconducting coherence length $\xi .5$ In contrast to that, there is no optimum size for the electromagnetic pinning as discussed in Refs. 6-9. Lithographically introduced micrometer size holes are proved to be efficient pinning centers for vortices in conventional superconductors, ${ }^{10,11}$ whereas nanometer columnar defects are more suitable for high- $T_{c}$ superconductors. ${ }^{5}$ Further improvement of the pinning properties can be achieved if besides the type and size of individual pinning centers, the geometrical distribution of pinning sites is also considered. ${ }^{8,12-16}$

An alternative way of creating attractive potential wells for vortices can be realized by exploiting the magnetic interaction between vortices and nanomagnets. The pioneer work of Alden and Livingston ${ }^{17}$ showed that embedded magnetic particles can indeed give rise to a clear enhancement of the critical currents. This early report has been later on corroborated by experiments carried out on other compounds with a better control of the mixing of nanoparticles into the superconducting matrix. ${ }^{18-29}$ More recently, it has been shown that the vortex structure can be modified due to the interaction between vortices and a nonuniform magnetic field generated at the surface of the superconductor. ${ }^{30,31}$ An effective modulation of the magnetic field at the surface has been achieved with magnetic particles deposited in a Bitter decoration experiment $^{32}$ and by the movement of a Bloch wall in a ferrite garnet film in contact with the superconductor. ${ }^{33}$ On the other hand, the fast development of the lithographic techniques made it possible to tailor, practically at will, any desired magnetic landscape at the sample surface. ${ }^{34-43}$ In particular, it has been shown that single $36,37,41,43-46$ and multiply ${ }^{47,48}$ connected structures of magnetic material can act as efficient on/off switchable pinning potential for vortices. The properties of this kind of pinning potential have been explored mainly through macroscopic measurements such as electrotransport and magnetization. Unfortunately, these integrated response techniques conceal the ultimate details of the microscopic world of vortex pinning.

In this work we present results on direct visualization of vortex patterns in a superconducting $\mathrm{Pb}$ film deposited on top of a periodic array of square-ring-shaped micromagnets. Real-space vortex arrangements are obtained in this type of hybrid system by combining two noninvasive highresolution imaging techniques: Bitter decoration and scanning Hall-probe microscopy (SHPM). The Bitter decoration experiments have revealed that the strong stray field generated by the square rings in the magnetized state acts as a funnel for the decoration particles, thus impeding the visualization of vortices which produce a much weaker local magnetic field. In addition, the inevitable polarization of the evaporated magnetic clusters due to the external field gives rise to a field-polarity-selected decoration process. One way to overcome these limitations is to image the vortices with SHPM. Images taken with this technique at $4.2 \mathrm{~K}$ unveil the actual location of vortices in our superconductor/ferromagnet hybrid system both in the as-grown and the dipolar states.

Previously, vortex imaging in a superconductor/ ferromagnet hybrid shows a preferential location of vortices at magnetic poles of opposite polarity (compensation effect).$^{38}$ In contrast, in our samples vortices sit at the mag- 
netic poles with the same polarity. This switching of the pinning potential can be due to the different number of vortex-antivortex $(\mathrm{V}-\mathrm{AV})$ pairs generated by the magnetic template, as anticipated in Ref. 49.

\section{EXPERIMENTAL DETAILS}

The samples studied in this work consist of a square array (4 $\mu \mathrm{m}$ period) of Co square rings fabricated by electronbeam (e-beam) lithography and subsequent lift-off technique. The density of vortices equals the density of square rings at a matching field of 1.3 Oe. The square rings are $250 \mathrm{~nm}$ wide, $25 \mathrm{~nm}$ thick, and have a lateral size of $2 \mu \mathrm{m}$. In order to avoid proximity effects, an insulating buffer layer of 10nm-thick Ge is deposited on the magnetic array. On top of that, a $50 \mathrm{~nm}$ layer of $\mathrm{Pb}$ is evaporated.

The electrical transport properties of the sample were studied using a Quantum Design cryostat with conventional electronics. For these measurements the Pb film was evaporated onto a predefined photoresist mask patterned into a transport bridge aligned with one of the principal axes of the square lattice of the square microrings. An ac current of $10 \mu \mathrm{A}$ with a frequency of $33 \mathrm{~Hz}$ was fed to the sample with a Keithley-6221 current source and the voltage was recorded using a SR-7265 lock-in amplifier. The temperature stability was within $1 \mathrm{mK}$.

The Bitter decoration experiments were performed at 4.2 $\mathrm{K}$ after cooling down the sample from above the critical temperature, $T_{c}$, in the presence of an applied magnetic field perpendicular to the sample surface [field cooling (FC) experiments]. In this way a homogeneous vortex distribution all over the sample is expected. The scanning Hall-probe microscopy images were obtained using a modified lowtemperature scanning Hall-probe microscope (LTSHPM) from Nanomagnetics Instruments. A temperature sensor and a heater were attached to the sample holder allowing fast stabilization and precise measurement of the sample temperature. As in the case of the Bitter decorations, the SHPM images were recorded at $4.2 \mathrm{~K}$ after FC the sample. The maximum scan area at this temperature is $\sim 13 \times 13 \mu \mathrm{m}^{2}$. The Hall voltage was measured in ac mode with a lock-in amplifier at a frequency of $5600 \mathrm{~Hz}$ and the rms value of the Hall current was $40 \mu \mathrm{A}$. The images were recorded in liftoff mode with the Hall sensor at a distance of $\sim 1 \mu \mathrm{m}$ from the surface of the sample. ${ }^{50}$

\section{TRANSPORT PROPERTIES}

In order to characterize the influence of the array of Co square rings on the superconducting properties of the $\mathrm{Pb}$ film, we explored the field and temperature dependence of the resistance, $R(H, T)$. The normal-metal/supercondutor $(\mathrm{N} /$ $\mathrm{SC})$ phase boundaries of the $\mathrm{Pb}$ with the Co square microrings in the as-grown and dipolar state are shown in Fig. 1 together with the results obtained in a coevaporated plain $\mathrm{Pb}$ film. The N/SC phase boundary lines were determined by $50 \%$ of the normal-state resistance.

The dipolar state, also known as the onion state, ${ }^{51}$ is the remanent state after applying a 3000 Oe in-plane field along

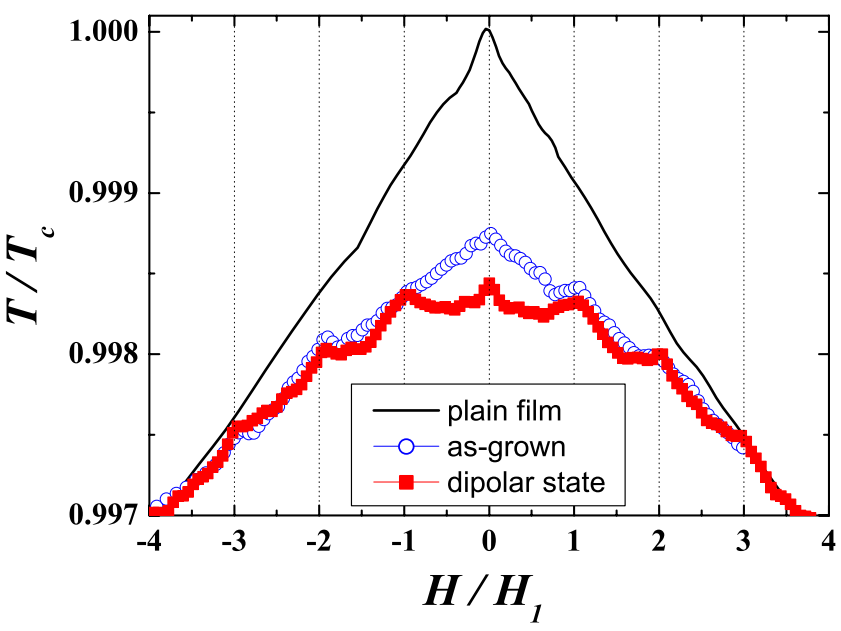

FIG. 1. (Color online) Normal/superconductor phase boundary for a plain $\mathrm{Pb}$ film (line) and $\mathrm{a} \mathrm{Pb}$ film on top of a square array of Co square microrings in the as-grown state (open circles) and in the dipolar state (solid squares). The temperature is normalized by $T_{c}$ $=7.2 \mathrm{~K}$ and the field by the matching field $H_{1}=1.3 \mathrm{Oe}$.

one diagonal of the square rings. In this state, head-to-head and tail-to-tail domain walls are formed in two opposite corners of the square rings. Figures 2(a) and 2(b) show, respectively, two-dimensional (2D) and three-dimensional (3D) plots of the out-of-plane component of the field, $B_{z}$, generated by an individual square ring in the dipolar state obtained by micromagnetic simulation. ${ }^{52}$ In the simulation we consider a Co square ring with the same dimensions as the one used in the experiment and a saturation magnetization $M_{s}$ $=140 \mathrm{emu} / \mathrm{cm}^{3}$. In the 2D plot [Fig. 2(a)] the red/blue (light gray/dark gray) contrast corresponds to positive and negative intensities of the field, respectively, and the arrows indicate the direction of the in-plane magnetization. The stray field is maximum (either with positive or negative sign) at the external part of the corners where the magnetic moments are head to head and tail to tail [see Fig. 2(b)]. In the other two corners there is a small modulation of the field produced by a gentle gradient of the magnetization.

The N/SC phase boundary, $H_{c 2}(T)$, of the plain film is linear (see the solid line in Fig. 1) as predicted from the Ginzburg-Landau equations $H_{c 2}=\left[\Phi_{0} / 2 \pi \xi^{2}(0)\right]\left(1-T / T_{c}\right){ }^{1}$
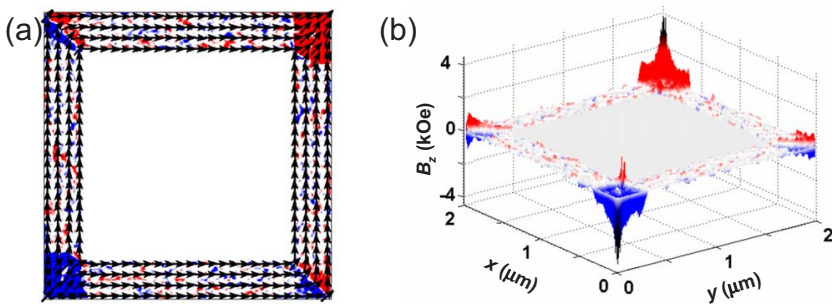

FIG. 2. (Color online) (a) 2D and (b) 3D plots of the out-ofplane field, $B_{z}$, generated by one magnetic square ring in the dipolar state obtained by micromagnetic simulation (Ref. 52). The dimensions of the square ring are the same as in the experiments and the values of the field are calculated at the surface of the square ring. The arrows in (a) indicate the direction of the in-plane magnetic moment. 
From the slope of this boundary we estimate the coherence length for our samples $\xi(0)=45.5 \mathrm{~nm}$. Since the BCS coherence length for $\mathrm{Pb}$ is $\xi_{0} \approx 83 \mathrm{~nm}$, we can use the dirty limit expression $\xi(0)=0.855 \sqrt{\xi_{0} l}$ to find the electronic mean-free path $l$. Using $l=34.1 \mathrm{~nm}$ and considering a renormalization ${ }^{53}$ of the penetration length due to the suppression of superconductivity induced by the magnetic array, we obtain $\lambda(0)$ $\approx 40.5 \mathrm{~nm}$. This calculation indicates that our $\mathrm{Pb}$ films are type-II superconductors. This is further confirmed by the detection of single-quanta vortices as observed by Bitter decorations of our samples.

As a consequence of the stray fields generated by the square microrings in the as-grown state the critical temperature, $T_{c}(H=0)$, of the sample grown on top of the array of magnetic square microrings is lower as compared to the one of the plain film. When the square rings are magnetized in the dipolar state the larger stray fields of the dipoles can locally destroy the superconducting condensate, inducing a further decrease in $T_{c}$. In this state, commensurability effects are observed at $\pm H_{1}$ and $\pm 2 H_{1}$. It is interesting to note that at $\pm 3 H_{1}$ the N/SC phase boundary lines of the plain film and the dipolar state merge together. This feature was also observed in $\mathrm{Al}$ samples deposited on top of Py square rings. ${ }^{47}$ From this result we can estimate the number of V-AV pairs generated by the stray field of the square rings in the dipolar state. Let us assume that $n_{p} \mathrm{~V}-\mathrm{AV}$ pairs per unit cell are created by the square microring array. When $H=n_{p} H_{1}$ all induced antivortices will be compensated by the vortices generated by the applied field. In this case the resultant vortex state consists of $n_{p}$ vortices induced by the square rings, which is equivalent to applying a field $H=n_{p} H_{1}$ in the plain film (where no V-AV pairs can be created). From that field on, i.e., $H= \pm 3 H_{1}$ in our particular case, the phase boundary of the plain film and the magnetized sample should overlap as indeed observed. This finding suggests that the estimated number of $\mathrm{V}-\mathrm{AV}$ pairs generated by the square rings is three. ${ }^{54}$

\section{BITTER DECORATION RESULTS}

The transport measurements presented above show that the array of Co square microrings acts as an effective magnetic pinning potential for vortices in the superconductor as long as $T \leqq T_{c}$. In order to gain information about the vortexmagnetic dipole interaction we performed Bitter decoration experiments at $4.2 \mathrm{~K}$. One of the main advantages of this technique is the possibility to visualize the distribution of vortices, with single vortex resolution, all over the surface of the sample (of the order of $\mathrm{mm}^{2}$ ).

As-grown state-a FC Bitter decoration experiment carried out at $4.2 \mathrm{~K}$ in the as-grown state at $H=0$ reveals the presence of a disordered state with about $45 \%$ of the square rings in a flux-closure state [see Fig. 3(a)]. This particular magnetic state is characterized by a small out-of-plane component of the stray field, and therefore, no agglomeration of decoration particles is observed on top of these square rings [Figs. 3(b) and 3(e)]. ${ }^{55}$ In the case of decorated square rings a variety of situations appears; the decoration particles accumulate in opposite corners of the square rings, indicating that

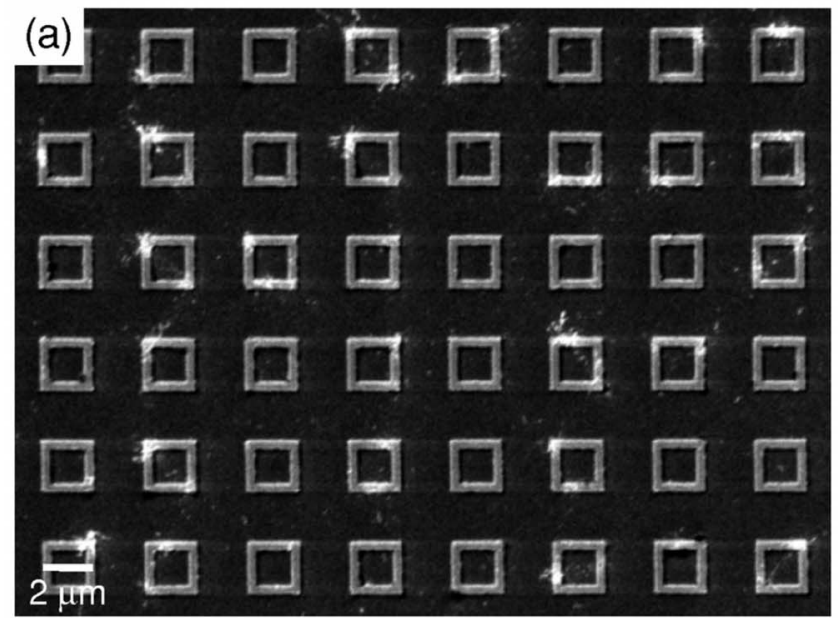

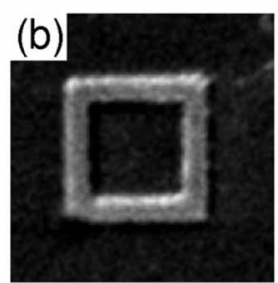

(e)

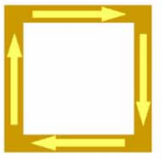

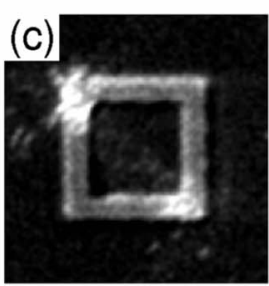

(f)

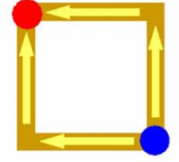

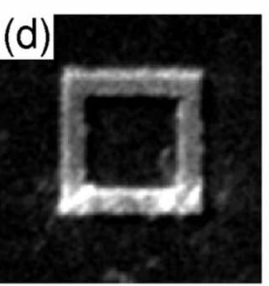

(g)

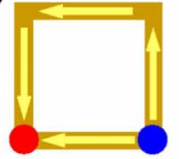

FIG. 3. (Color online) (a) Scanning electron microscope (SEM) image of a Bitter decoration performed in the as-grown state at $H$ $=0$ and $T=4.2 \mathrm{~K}$. Zoom in of panel (a) at the particular square rings where a (b) flux-closure, (c) dipolar, and (d) horseshoe states are observed. [(e)-(g)] Schematics of a square ring in three different states shown in (b)-(d), respectively. The yellow (light gray) arrows indicate the average direction of the in-plane magnetization. The red (gray) and blue (black) dots in (f) and (g) denote the positive and negative magnetic poles, respectively.

those loops are in a dipolar state [Figs. 3(c) and 3(f)] (Ref. 56) or neighboring corners appear decorated in square rings that are in the so-called horseshoe state ${ }^{47}$ [Figs. 3(d) and $3(\mathrm{~g})]$. The horseshoe state is such that the net dipolar moment is parallel to the side of the square ring that connects the two decorated corners. In addition, in some cases a single spot along one side or at one corner of the square rings is decorated. This could correspond to the presence of magnetic vortices that generate an out-of-plane field in their center. ${ }^{57}$

Magnetized state-Fig. 4(a) shows a FC decoration image at $H=0$ after the square rings have been magnetized by applying a uniform in-plane field of 3000 Oe parallel to one diagonal of the square rings [see the white arrow in Fig. 4(c)]. As expected, the magnetic dipoles generated by the stray fields have an average magnetic moment along this diagonal, resulting in two intercalated square lattices of positive and negative out-of-plane fields. Since the decoration is made on the surface of the superconductor, one would expect that the decorated poles correspond to vortices and antivortices located at the corners of the square rings. The same 

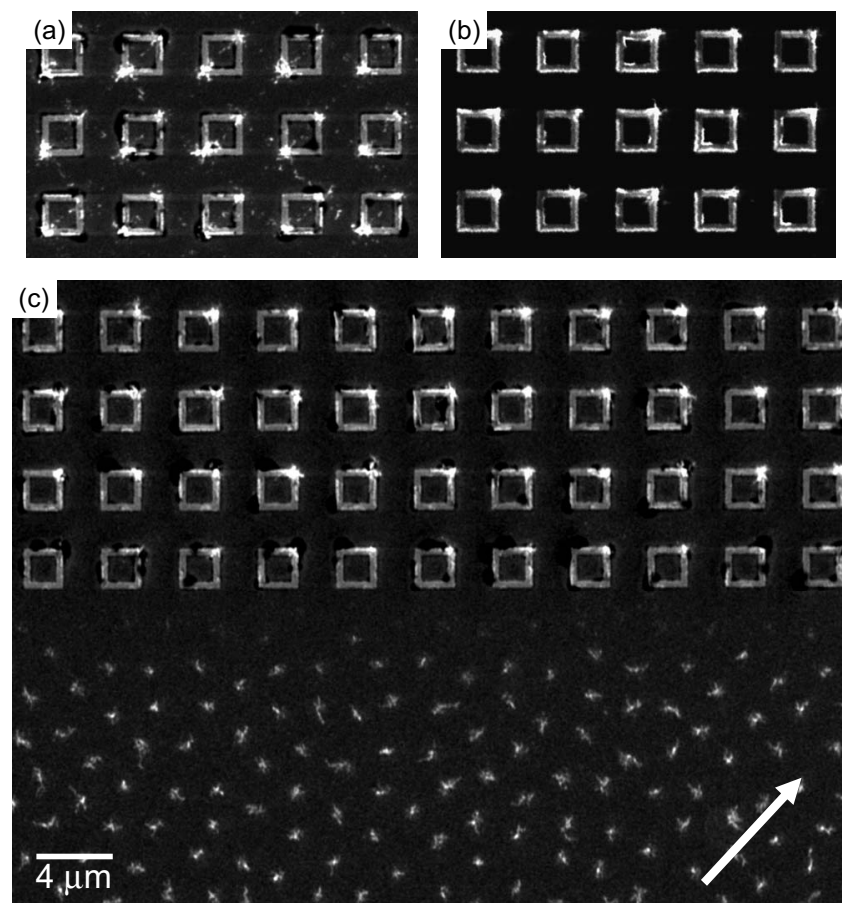

FIG. 4. Bitter decoration with Co square rings magnetized in the dipolar state at $T=4.2 \mathrm{~K}$ and (a) $H=0$, (b) $H=H_{1}$, and (c) $H$ $=3 H_{1}$. In the last case the image is taken at the boundary between the patterned and nonpatterned regions. The white arrow indicates the direction of the in-plane field used to magnetize the square rings.

result, namely, the decoration of the corners of the ring where the stray field is maximum, is obtained when decorating directly on top of the Co square rings without the $\mathrm{Pb}$ on top (not shown).

A possible way to evidence the influence of the SC layer consists in applying a finite field in such a way that both the magnetic poles due to the square rings and the vortices are decorated independently. Figure 4(b) shows the result of a magnetic decoration performed at $H=H_{1}$. Strikingly, the image shows that only one corner (upper right) of the square rings is decorated. This result can be in principle attributed to the fact that vortices generated by the applied field sit at the positions where the out-of-plane field of the dipole is negative. ${ }^{38}$ Then, the decoration particles accumulate on the corners of the square rings where the field of the dipoles is not compensated. Under this circumstance it is expected that for high enough fields (i.e., strictly speaking $H>n_{p} H_{1}$ ), interstitial vortices would appear and agglomeration of decoration particles should locate in between the square rings. However, decorations performed at higher applied fields, $H$ $=3 H_{1}$ and $H=10 H_{1}$, still show that only the upper-right corners of the square rings are decorated and no interstitial vortices are observed. A similar result is obtained in the case of a decoration performed at $H=0.5 H_{1}$. As an example, Fig. 4(c) shows an image taken in the neighborhood of the boundary between the patterned and nonpatterned regions in a decoration experiment made at $H=3 H_{1}$. As expected, in the nonpatterned region vortices form a distorted triangular array with a density corresponding to the applied field.
Notice that there are two important points in these results. First, the lack of interstitial vortices in the case $H>n_{p} H_{1}$ and second the decoration of a single magnetic pole for decorations made at $H \neq 0$. In order to understand the origin of the observed lack of interstitial vortices it is necessary to compare the field generated by the magnetic dipoles with that associated with vortices. On the surface of the sample, the field generated at the center of the vortex is approximately ${ }^{1}$

$$
2 H_{c 1}=\frac{\Phi_{0}}{2 \pi \lambda^{2}} \ln (\lambda / \xi),
$$

where $\Phi_{0}$ is the flux quantum. Using the values of $\lambda$ and $\xi$ estimated for our sample we find that the maximum field generated by a vortex at the surface of the sample at $4.2 \mathrm{~K}$ is about 200 Oe. On the other hand, from the micromagnetic simulations we can estimate the stray field induced by the square rings at the surface of the superconductor (this corresponds to a distance of $50 \mathrm{~nm}$ above the square ring surface). This calculation shows that the maximum out-of-plane field (at the corner of the square ring) is $\sim 1000$ Oe, i.e., five times larger than the field of a vortex. Consequently, in a decoration experiment the evaporated magnetic particles "feel" the dipolar field that acts as a funnel accumulating most of the particles at the positions of the poles preventing the decoration of interstitial vortices. This is confirmed by the decoration at the border of the pattern shown in Fig. 4(c). At first sight it seems that there is a region free of vortices right next to the first row of Co square rings. However, a close inspection of the image shows a row of decoration clumps in that area much less brighter than the rest. Vortices that are at a distance of the order of $4 \mu \mathrm{m}$ from the edge of the pattern are decorated in a normal way. This indicates that one magnetic pole can attract magnetic particles that are at a maximum distance of $\sim 6 \mu \mathrm{m}$ (i.e., larger than the separation between magnetic poles).

The issue that still remains unsettled is why only one pole of the square rings is decorated when a finite magnetic field is applied. This observation can be ascribed to the fact that the magnetic moment of the evaporated particles aligns with the direction of the applied field at a distance quite far away from the sample surface as schematically represented in Fig. 5 . When clusters of polarized particles interact with the dipolar field generated by the square microrings, they are attracted to the pole with parallel field orientation and repelled by the other one. Consequently, the absence of decoration particles on the lower-left corner of the square rings is not necessarily due to the presence of vortices but due to the broken symmetry imposed by the applied magnetic field. This is confirmed by the result of a decoration experiment in a sample of Co square rings without a superconducting layer on top. In this case we perform the decoration with the square rings in the dipolar state and a perpendicular applied field of 10 Oe. One would expect that in this situation both poles will appear equally decorated since the applied field is not strong enough to modify the field distribution generated by the square rings. However, it is observed (not shown) that the decoration particles only accumulate in the corners of the square rings where the stray field is parallel to the applied 


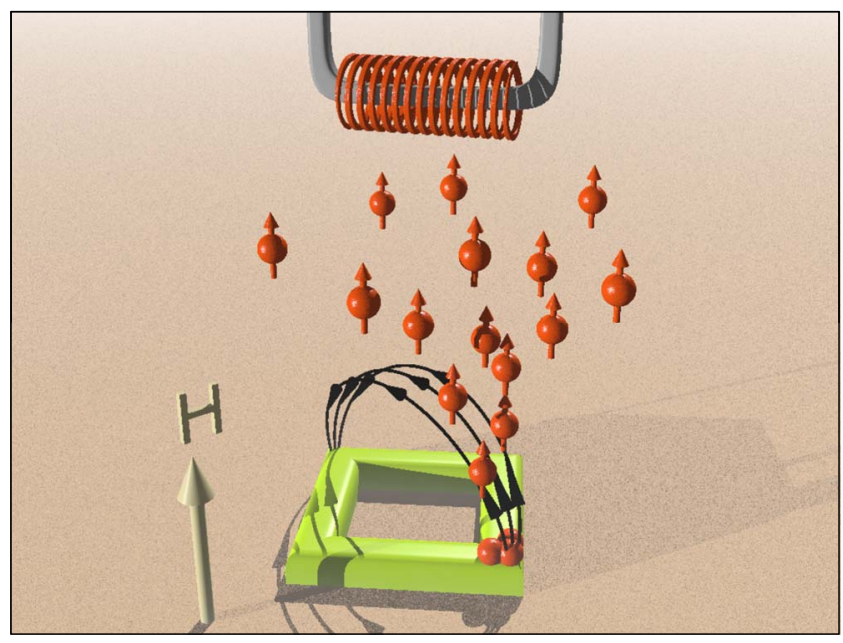

FIG. 5. (Color online) Schematic presentation of the accumulation of polarized decoration particles on top of one corner of the Co square microrings (see text). The black arrows indicate the stray field induced by the magnetized square ring while the gray arrow denotes the direction of the applied field. On the upper part of the image the filament that generates the decoration particles (red spheres) is shown.

field. This finding suggests that it is not possible to decorate either vortices or the stray field generated by magnetic structures when the local magnetic field direction is opposite to the one of the applied field.

\section{SCANNING HALL-PROBE MICROSCOPY RESULTS}

As-grown state-a possible way to overcome this inherent limitation of the Bitter decorations can be achieved by using a less invasive high-resolution technique: SHPM. Figure 6(a) shows an image obtained by SHPM after FC a sample with a square array of Co square rings in the asgrown state in a field $H \approx 1.5 H_{1}$. In the lower-left part of the image there is a bright and a dark spot next to each other (see circles in the image). In addition, over the right edge of the picture there is also a dark spot. These features appear in an image taken at zero applied field [Fig. 6(b)] as well as in images taken at different applied fields, indicating that they correspond to the out-of-plane stray field generated by the square microrings. After identifying the location of the square rings ${ }^{58}$ (dotted squares in the image), it is possible to see that the pair of bright and dark spots is due to the stray field of a square ring in a horseshoe state [similar to the case shown in Fig. 3(d)]. Due to the large intensity difference between the field emanating from the magnetic poles and that produced by the vortices, it is difficult to identify flux lines directly in the image. Therefore, in order to determine the location of vortices it is necessary to remove the contribution of the field from the square rings. This is achieved by subtracting from the raw images the field intensity picture taken at $H=0$ [see Fig. 6(b)] where no vortices induced by the applied field are present. The result of this subtraction is shown in Fig. 6(c) where the vortices are seen as bright spots. We identify the local maxima of intensity as the vortex positions and depicted them as black dots in Fig. 6(d). In most of the cases, vortices sit at the corners of the square rings, forming a rather disordered structure. In the case of the square ring in the horseshoe state a vortex sits along the side of the square ring in between the two poles. It is interesting to note that the values of the magnetic induction of the raw image spans from -10 to $6 \mathrm{G}$ [see the scale bar of Fig. 6(a)], while after the subtraction of the $H=0$ image the range of intensity is limited to the range $2.5-8 \mathrm{G}$ [Fig. 6(c)]. This difference in scale of magnetic field intensity is due to the stronger field induced by the square microrings as compared to the weaker field emanating from the vortices.

Magnetized state - a different situation appears after magnetizing the sample by applying an in-plane field of $3000 \mathrm{Oe}$ parallel to one of the diagonals of the square rings, see arrow in Fig. 6(e). In this case a periodic array of magnetic dipoles is formed, as clearly seen in the image shown in Fig. 6(e) obtained after FC the sample down to $4.2 \mathrm{~K}$ in a field $H$ $\approx 1.5 H_{1}$.

Similar to the case of the as-grown sample, a subtraction of the $H=0$ image [Fig. 6(f)] is necessary in order to identify vortex positions [see Fig. 6(g)]. From the field induction scale bars of Figs. 6(e) and 6(g) we can see that the intensity of the dipolar fields is approximately five times larger than the vortex field intensity, in good agreement with the estimation made in Sec. IV.

In Figs. 6(h)-6(j) the vortex distributions at $H \approx 1.5 H_{1}$, $H \approx-1.5 H_{1}$, and $H \approx 3 H_{1}$, respectively, are shown. The location of the centers of the positive and negative magnetic poles is indicated by white circles and crosses, respectively. The results of our SHPM experiments show that in the case of FC in a positive field $\approx 1.5 H_{1}$, most of the vortices locate on top of the positive poles [Fig. 6(h)]. The fact that not all of the positive poles are occupied could be due to the influence of intrinsic pinning or due to small inhomogeneities in the sample. When the sign of the field is reversed most of the vortices (with negative polarity) sit on top of the negative poles [Fig. 6(i)].

Previous SHPM studies on Pb samples deposited on top of an array of $\mathrm{Co} / \mathrm{Pt}$ dots with out-of-plane magnetization evidence that vortices sit mainly at positions where the stray field of the micromagnet is parallel to the vortex field. ${ }^{40}$ This behavior could be understood considering that the dots generate a pinning potential for vortices,

$$
U_{m}(\mathbf{r})=-\int_{V_{f}} \mathbf{m}\left(\mathbf{r}^{\prime}\right) \cdot \mathbf{B}\left(\mathbf{r}-\mathbf{r}^{\prime}\right) d \mathbf{r}^{\prime},
$$

where $\mathbf{m}$ is the magnetization of the dot, $\mathbf{B}$ is the field of the vortex, and the integration is done over the volume of the ferromagnet. This potential has a minimum on top of the dots when the applied field $H$ (and consequently the field of the vortices) is parallel to the magnetization direction. On the other hand, similar experiments in $\mathrm{Pb}$ samples with arrays of bars with in-plane magnetization show ${ }^{38}$ also field-polaritydependent pinning but now due to the interaction between vortices and vortex-antivortex pairs generated by the magnetic bars. In this case vortices sit at the poles where the stray field is opposite to the applied field. Theoretical calcu- 


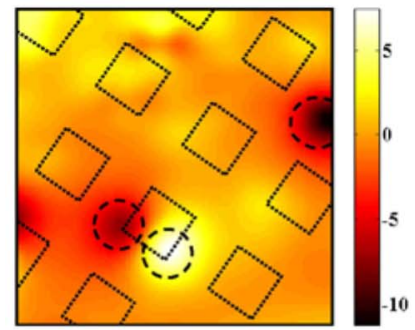

(a) $H / H_{1} \approx 1.5$

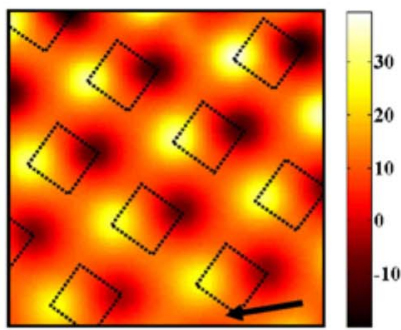

(e) $H / H_{1} \approx 1.5$

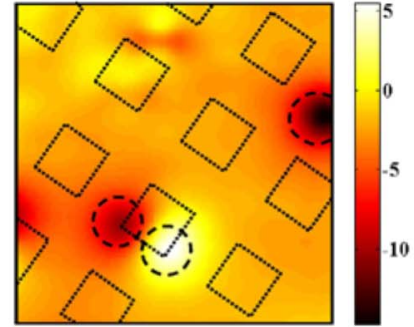

(b) $H / H_{1}=0$

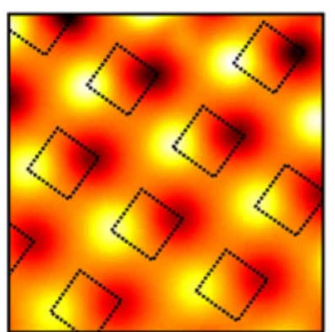

(f) $H / H_{1}=0$

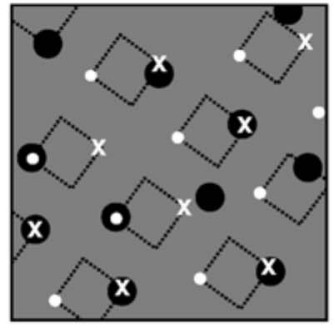

(i) $H / H_{1} \approx-1.5$

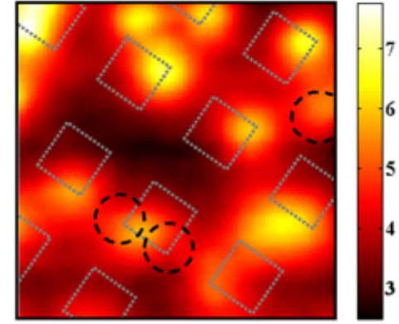

(c) $H / H_{1} \approx 1.5$

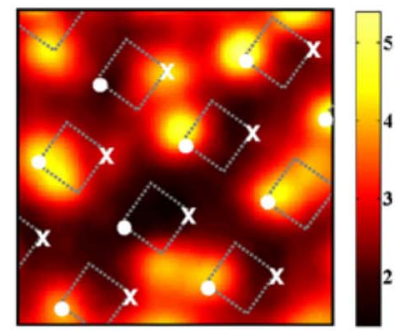

(g) $H / H_{1} \approx 1.5$

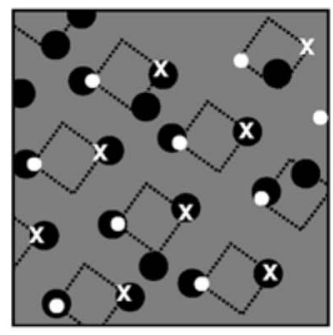

(j) $H / H_{1} \approx 3$

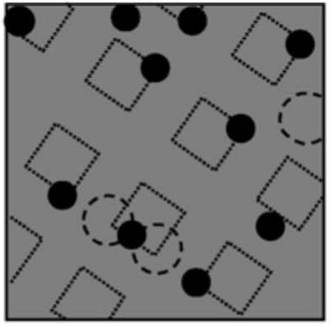

(d) $H / H_{1} \approx 1.5$

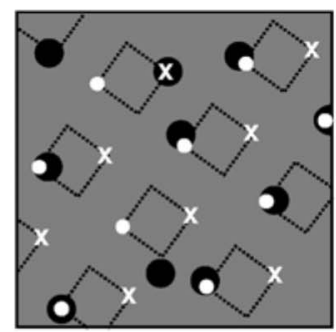

(h) $H / H_{1} \approx 1.5$

FIG. 6. (Color online) [(a) and (b)] Raw SHPM images taken after FC down to $T=4.2 \mathrm{~K}$ in a field $H \approx 1.5 H_{1}$ and $H=0$, respectively, with the square rings in the as-grown state. The circles indicate regions of strong stray fields generated by the square rings that are in a magnetized state. (c) Vortex image in the as-grown state at $H \approx 1.5 H_{1}$. This image is obtained by subtracting the image (b) $(H=0)$ from (a). In (d) the black dots indicate the vortex locations that were identified as local maxima in (c). [(e) and (f)] Raw images taken after FC in a field $H \approx 1.5 H_{1}$ and $H=0$, respectively, with the square rings in the dipolar state. (g) Vortex image at $H \approx 1.5 H_{1}$ obtained by subtracting (f) from (e). Vortex location in the dipolar state at $(\mathrm{h}) H \approx 1.5 H_{1}$, (i) $H \approx-1.5 H_{1}$, and (j) $H \approx 3 H_{1}$. In all images the dotted lines indicate the positions of the square rings, the field of view is $12.6 \times 12.6 \mu \mathrm{m}^{2}$ and the color (gray) scale bars correspond to the magnetic induction in units of Gauss. In (e) the black arrow indicates the orientation of the in-plane field used to magnetize the square microrings.

lations of the interaction between vortices and a ferromagnet with in-plane magnetization demonstrate ${ }^{49}$ that whether the vortex will be pinned by one or the other pole of the micromagnet depends on the strength of the micromagnet magnetization. In the case where the magnetization is large enough to induce a vortex-antivortex pair in the superconductor, the equilibrium position of a vortex generated by an applied field is the result of the balance among vortex-micromagnet, vortex-vortex, and vortex-antivortex interactions. It is important to mention that in the calculations only the interaction of a single vortex with one magnetic microstructure that can generate, at maximum, one vortex-antivortex pair is considered. Unfortunately, so far there is no prediction of the lowest-energy vortex state considering also the interaction with other vortices generated by the applied field and with more than one induced vortex-antivortex pair. In our experiments we observe that at low densities vortices sit mostly on top of the corners of the square rings [Figs. 6(h) and 6(i)]. Therefore we can assure that the magnetic square microrings act as effective pinning centers for vortices even when lowering the temperature down to $4.2 \mathrm{~K}$.

Finally, when the field is further increased, SHPM FC experiments show that vortices start to locate also at the negative poles of the micromagnets, see, for example, the vortex distribution at $H \approx 3 H_{1}$ shown in Fig. 6(j). Few vortices sit at the corners of the square rings where the out-ofplane field is very small (for example, see the square microring at the upper-left corner of the image) but no vortices are seen to locate in the interstitial sites in between the square rings. A possible explanation for the observed vortex configuration is that vortices placed at the positive poles generate a caging potential ${ }^{59}$ with a minimum in the location of the negative magnetic poles. As a counterpart, the force exerted on the vortices by the negative magnetic poles [see 
Eq. (2)] would tend to repel vortices from those positions. Thus, the observed vortex distribution at higher densities seems to be a result of the balance between vortex-vortex and vortex-magnetic pole interactions. Our SHPM experiments in the magnetized state show that the corners of the square rings, both with magnetization parallel and opposite to the vortex field, represent effective pinning sites for vortices.

\section{CONCLUSIONS}

We have studied the influence of an array of Co square microrings on the superconducting properties of a $\mathrm{Pb}$ film. From transport measurements we observe that the stray fields induced by the magnetic loops cause a reduction in $T_{c}$ with respect to a plain $\mathrm{Pb}$ film, in agreement with earlier reports. Furthermore, when the square rings are in a dipolar state, matching features are observed. This indicates that the micromagnets act as pinning centers for vortices near the N/SC phase boundary line. On the other hand, Bitter decorations at 4.2 $\mathrm{K}$ in the as-grown state show that almost half of the square rings are in a flux-closure state. In the dipolar state, the strong stray magnetic field associated with the square rings act as funnels for the magnetic decoration particles. Due to this effect, the particles accumulate on the corners of the square rings, preventing the decoration of vortices. Our results also suggest that it is not possible to decorate regions where the local magnetic field direction is opposite to the one of the applied field. SHPM experiments in the as-grown state show that at low temperatures, the square rings act as pinning centers even if they are in a flux-closure state. Besides, the images obtained in FC experiments confirm that the field induced by the square rings in the dipolar state is much larger than the field of the vortices. This result is consistent with the results obtained by micromagnetic simulations. Moreover, FC images taken in the dipolar state and at low vortex densities show that the poles with magnetization parallel to the applied field create strong pinning sites for vortices. At higher fields, the opposite poles can also act as preferential sites for vortices, suggesting that the resultant vortex structure is a consequence of the competition between vortex-vortex and vortex-magnetic dipole interactions. Our results indicate the necessity of a theoretical model of a superconductor/ferromagnet hybrid system which takes into account vortex-vortex interactions and the interaction of a vortex with more than one induced vortex-antivortex pair.

\section{ACKNOWLEDGMENTS}

We would like to thank F. de la Cruz, Y. Fasano, R. Luccas, P. Mispelter, and P. De Greef for useful technical discussions and W. Gillijns for a careful reading of the paper. This work was supported by the Research Fund K.U. Leuven under Grant No. GOA/2004/02, the Fund for Scientific Research-Flanders FWO-Vlaanderen, the Belgian InterUniversity Attraction Poles IAP, the European ESF NES programs, the U.S. NSF under Grant No. ECCS-0823813, and CNM ANL under Grants No. Nr.468 and Nr.470. A.V.S. is grateful for the support from the FWO-Vlaanderen.
${ }^{1}$ M. Tinkham, Introduction to Superconductivity, 2nd ed. (McGraw-Hill, New York, 1996).

${ }^{2}$ A. E. Campbell and J. E. Evetts, Adv. Phys. 21, 199 (1972).

${ }^{3}$ G. Blatter, M. V. Feigelman, V. B. Geshkenbein, A. I. Larkin, and V. M. Vinokur, Rev. Mod. Phys. 66, 1125 (1994).

${ }^{4}$ A. Buzdin and D. Feinberg, Physica C 235-240, 2755 (1994).

${ }^{5}$ L. Civale, A. D. Marwick, T. K. Worthington, M. A. Kirk, J. R. Thompson, L. Krusin-Elbaum, Y. Sun, J. R. Clem, and F. Holtzberg, Phys. Rev. Lett. 67, 648 (1991).

${ }^{6}$ G. S. Mkrtchyan and V. V. Shmidt, Sov. Phys. JETP 34, 195 (1972).

${ }^{7}$ N. Takezawa and K. Fukushima, Physica C 228, 149 (1994).

${ }^{8}$ V. V. Moshchalkov, M. Baert, V. V. Metlushko, E. Rosseel, M. J. Van Bael, K. Temst, Y. Bruynseraede, and R. Jonckheere, Phys. Rev. B 57, 3615 (1998).

${ }^{9}$ H. Nordborg and V. M. Vinokur, Phys. Rev. B 62, 12408 (2000).

${ }^{10}$ A. Bezryadin and B. Pannetier, J. Low Temp. Phys. 98, 251 (1995).

${ }^{11}$ V. V. Moshchalkov, M. Baert, V. V. Metlushko, E. Rosseel, M. J. Van Bael, K. Temst, Y. Bruynseraede, and R. Jonckheere, J. Phys. IV 6, C3-335 (1996).

${ }^{12}$ C. Reichhardt and C. J. Olson Reichhardt, Phys. Rev. B 76, 094512 (2007).

${ }^{13}$ M. Kemmler, C. Gurlich, A. Sterck, H. Pohler, M. Neuhaus, M. Siegel, R. Kleiner, and D. Koelle, Phys. Rev. Lett. 97, 147003
(2006).

${ }^{14}$ J. E. Villegas, M. I. Montero, C.-P. Li, and Ivan K. Schuller, Phys. Rev. Lett. 97, 027002 (2006).

${ }^{15}$ V. Misko, S. Savel'ev, and F. Nori, Phys. Rev. Lett. 95, 177007 (2005).

${ }^{16}$ A. V. Silhanek, W. Gillijns, V. V. Moshchalkov, B. Y. Zhu, J. Moonens, and L. H. A. Leunissen, Appl. Phys. Lett. 89, 152507 (2006).

${ }^{17}$ T. H. Alden and J. D. Livingston, Appl. Phys. Lett. 8, 6 (1966).

${ }^{18}$ A. Snezhko, T. Prozorov, and R. Prozorov, Phys. Rev. B 71, 024527 (2005).

${ }^{19}$ N. D. Rizzo, J. Q. Wang, D. E. Prober, L. R. Motowidlo, and B. A. Zeitlin, Appl. Phys. Lett. 69, 2285 (1996).

${ }^{20}$ J.-Q. Wang, N. D. Rizzo, D. E. Prober, L. R. Motowidlo, and B. A. Zeitlin, IEEE Trans. Appl. Supercond. 7, 1130 (1997).

${ }^{21}$ N. M. Suleimanov, P. N. Togulev, V. V. Bazarov, and I. B Khaibullin, Physica C 404, 363 (2004).

${ }^{22}$ D. Stamopoulos, M. Pissas, and E. Manios, Phys. Rev. B 71, 014522 (2005).

${ }^{23}$ D. Stamopoulos, E. Manios, M. Pissas, and D. Niarchos, Physica C 437-438, 289 (2006).

${ }^{24}$ T. Kuroda, T. Nakane, H. Uematsu, and K. Kumakura, Supercond. Sci. Technol. 19, 1152 (2006).

${ }^{25}$ P. N. Togoulev, N. M. Suleimanov, and K. Conder, Physica C 450, 45 (2006). 
${ }^{26}$ A. Palau, J. L. MacManus-Driscoll, and M. G. Blamire, Supercond. Sci. Technol. 20, S136 (2007).

${ }^{27}$ S. Haindl, M. Weisheit, V. Neu, L. Schultz, and B. Holzapfel, Physica C 463-465, 1001 (2007).

${ }^{28}$ A. Palau, H. Parvaneh, N. A. Stelmashenko, H. Wang, J. L. Macmanus-Driscoll, and M. G. Blamire, Phys. Rev. Lett. 98, 117003 (2007).

${ }^{29}$ S. Haindl, M. Weisheit, T. Thersle, L. Schultz, and B. Holzapfel, Supercond. Sci. Technol. 21, 045017 (2008).

${ }^{30}$ S. Erdin, I. F. Lyuksyutov, V. L. Pokrovsky, and V. M. Vinokur, Phys. Rev. Lett. 88, 017001 (2001).

${ }^{31}$ G. Carneiro, Phys. Rev. B 72, 144514 (2005); Physica C 432, 206 (2005).

${ }^{32}$ Y. Fasano, J. A. Herbsommer, F. de la Cruz, F. Pardo, P. L. Gammel, E. Bucher, and D. J. Bishop, Phys. Rev. B 60, R15047 (1999).

${ }^{33}$ P. E. Goa, H. Hauglin, A. A. F. Olsen, D. Shantsev, and T. H. Johansen, Appl. Phys. Lett. 82, 79 (2003).

${ }^{34}$ O. Geoffroy, D. Givord, Y. Otani, B. Pannetier and F. Ossart, J. Magn. Magn. Mater. 121, 223 (1993); Y. Otani,B. Pannetier, J. P. Nozieres, and D. Givord, ibid. 126, 622 (1993).

${ }^{35}$ J. I. Martín, M. Velez, J. Nogues, and I. K. Schuller, Phys. Rev. Lett. 79, 1929 (1997).

${ }^{36}$ M. J. Van Bael, K. Temst, V. V. Moshchalkov, and Y. Bruynseraede, Phys. Rev. B 59, 14674 (1999).

${ }^{37}$ L. van Look, M. J. van Bael, K. Temst, J. G. Rodrigo, M. Morelle, V. V. Moshchalkov, and Y. Bruynseraede, Physica C 332, 356 (2000).

${ }^{38}$ M. J. Van Bael, J. Bekaert, K. Temst, L. Van Look, V. V. Moshchalkov, Y. Bruynseraede, G. D. Howells, A. N. Grigorenko, S. J. Bending, and G. Borghs, Phys. Rev. Lett. 86, 155 (2001).

${ }^{39}$ M. Lange, M. J. Van Bael, Y. Bruynseraede, and V. V. Moshchalkov, Phys. Rev. Lett. 90, 197006 (2003).

${ }^{40}$ M. J. Van Bael, M. Lange, S. Raedts, V. V. Moshchalkov, A. N. Grigorenko, and S. J. Bending, Phys. Rev. B 68, 014509 (2003).

${ }^{41}$ J. E. Villegas, S. Savel'ev, F. Nori, E. M. Gonzalez, J. V. Anguita, R. Garcia, and J. L. Vicent, Science 302, 1188 (2003).

${ }^{42}$ Y. Fasano, M. De Seta, M. Menghini, H. Pastoriza, and F. de la Cruz, Solid State Commun. 128, 51 (2003).

${ }^{43}$ W. Gillijns, A. V. Silhanek, and V. V. Moshchalkov, Phys. Rev. B 74, 220509(R) (2006).

${ }^{44}$ J. E. Villegas, K. D. Smith, L. Huang, Y. Zhu, R. Morales, and I. K. Schuller, Phys. Rev. B 77, 134510 (2008).

${ }^{45}$ A. Hoffmann, L. Fumagalli, N. Jahedi, J. C. Sautner, J. E. Pearson, G. Mihajlovic, and V. Metlushko, Phys. Rev. B 77, 060506(R) (2008).

${ }^{46}$ J. S. Neal, M. V. Milošević, S. J. Bending, A. Potenza, L. San Emeterio, and C. H. Marrows, Phys. Rev. Lett. 99, 127001 (2007).

${ }^{47}$ A. V. Silhanek, N. Verellen, V. Metlushko, W. Gillijns, F.
Gozzini, B. Ilic, and V. V. Moshchalkov, Physica C 468, 563 (2008); A. V. Silhanek, W. Gillijns, V. V. Moshchalkov, V. Metlushko, and B. Ilic, Appl. Phys. Lett. 89, 182505 (2006).

${ }^{48}$ N. Verellen, A. V. Silhanek, V. Metlushko, W. Gillijns, F. Gozzini, B. Ilic, and V. V. Moshchalkov, Appl. Phys. Lett. 93, 022507 (2008).

${ }^{49}$ M. V. Milošević and F. M. Peeters, Phys. Rev. B 69, 104522 (2004).

${ }^{50}$ In our scanning Hall-probe microscope a scanning tunneling microscope (STM) tip and a Hall probe are integrated in the same chip. The STM tip is used to approach the Hall probe to the sample surface, i.e., the tip is in contact with the surface when a tunneling current is detected. This approach is made on top of the highest point of the sample (top-right corner in our images). In a lift-off mode operation the STM tip is retracted at a certain distance (lift-off distance) from the sample surface. Since the Hall probe is located $15 \mu \mathrm{m}$ behind the STM tip, the distance between the Hall probe and the sample surface is larger than the lift-off distance. In our case we estimate a tilt angle of $\sim 3^{\circ}$. Therefore, since during the experiments the lift off is $200 \mathrm{~nm}$ the distance between the Hall sensor and the sample is $\sim 1 \mu \mathrm{m}$ at the approaching point. This separation increases as the image is scanned, giving rise to a decrease in contrast in the images from top to bottom. For more details see S. J. Bending, Adv. Phys. 48, 449 (1999).

${ }^{51}$ X. B. Zhu, P. Grutter, V. Metlushko, and B. Ilic, J. Appl. Phys. 93, 7059 (2003).

${ }^{52}$ Micromagnetic simulations are performed with a public available code from NIST (http://math.nist.gov/oommf).

${ }^{53}$ A. Wahl, V. Hardy, J. Provost, Ch. Simon, and A. Buzdin, Physica C 250, 163 (1995).

${ }^{54}$ Note that this is an approximate estimate.

${ }^{55}$ The bright edges of the square microring shown in Fig. 3(b) are due to the charging effects during the acquisition of the image with SEM.

${ }^{56}$ In a decoration experiment regions with positive and negative out-of-plane local magnetic fields are not distinguished from each other since the decoration particles are sensitive to the field gradient independently of the field direction.

${ }^{57}$ A. V. Silhanek, W. Gillijns, V. V. Moshchalkov, V. Metlushko, F. Gozzini, B. Ilic, W. C. Uhlig, and J. Unguris, Appl. Phys. Lett. 90, 182501 (2007).

${ }^{58}$ In order to determine the location of the square rings an out-ofplane field of the order of 5000 Oe was applied after doing the FC experiments in the as-grown state. Subsequently a SHPM image was taken where the square rings appear as bright spots due to the finite out-of-plane component of the magnetization.

${ }^{59}$ I. B. Khalfin and B. Ya. Shapiro, Physica C 207, 359 (1993); E. Rosseel, M. Van Bael, M. Baert, R. Jonckheere, V. V. Moshchalkov, and Y. Bruynseraede, Phys. Rev. B 53, R2983 (1996). 